\title{
A nondestructive contactless technique to assess the viscoelasticity of blood clots in real-time
}

\author{
Shiva Naseri ${ }^{\mathrm{a}}$, Newsha Koushki ${ }^{\mathrm{b}}$, Ehsan Rezabeigi ${ }^{\mathrm{a}}$, Allen Ehrlicher ${ }^{\mathrm{b}}$, Showan N. Nazhat ${ }^{\mathrm{a},{ }^{*}}$ \\ ${ }^{\text {a }}$ Department of Mining and Materials Engineering, McGill University, Montreal, QC, Canada \\ ${ }^{\mathrm{b}}$ Department of Bioengineering, McGill University, Montreal, QC, Canada
}

\section{A R T I C L E I N F O}

\section{Keywords:}

Blood coagulation

Viscoelastic properties

TEG

Rheometer

Elastosens ${ }^{\mathrm{TM}} \mathrm{Bio}^{2}$

\begin{abstract}
A B S T R A C T
There is a need for reliable and quantitative real-time assessment of blood properties to study and treat a broad spectrum of disorders and cardiovascular diseases as well as to test the efficacy of hemostatic agents. In this study, the real-time changes in viscoelastic/rheological properties of bovine whole blood during coagulation induced by different concentrations of calcium chloride $\left(\mathrm{CaCl}_{2} ; 15,25,35\right.$ and $\left.45 \mathrm{mM}\right)$ was investigated. For this purpose, a novel, contactless technique was used to accurately measure the clotting characteristics under controlled and sterile conditions. It was demonstrated that, increasing the calcium concentration from low values (i.e., 15 and $25 \mathrm{mM}$ ), led to shorter reaction time; however, a further increase in calcium concentration (i.e., 35 and $45 \mathrm{mM}$ ) favored longer reaction times. Additionally, increasing the $\mathrm{CaCl}_{2}$ concentration resulted in higher shear storage modulus (i.e., stiffer clots). These results were also comparable to those generated by thromboelastrograph, a clinically established technique, as well as a conventional rheometer, which quantitatively verified the high correlation of the shear storage modulus data. In sum, the non-destructive testing technique used in this study is reproducible and sensitive in measuring clot formation kinetics, which could be applied to assess the efficacy of hemostatic agents, and may also contribute to better diagnosing relevant circulatory system diseases and conditions.
\end{abstract}

\section{Introduction}

Blood coagulation is a complex multi-scale process composed of three distinct stages (Guillin and Bezeoud, 1985; Tran et al., 2013; Weisel, 2010): 1) the blood is in a liquid state; 2) the blood progressively converts into a gel state due to the transformation of fibrinogen into fibrin through a coagulation cascade reaction; and 3) the clot reaches stability and maximum stiffness. In general, blood coagulation on synthetic material surfaces is initiated by a rapid deposition of a protein layer (i.e., fibrin deposition) (Tanaka et al., 2015; Berglin et al., 2004).

Blood biomechanics can be correlated with various diseases and lifelong genetic conditions affecting blood clotting, such as bleeding disorders, hemophilia, von Willebrand disease, rare factor deficiencies, and platelet function disorders (Peyvandi et al., 2006). Therefore, it is critical to accurately evaluate blood biomechanics, including changes in viscoelastic properties that can represent its stiffness values (Tran et al., 2013). There are various methods to evaluate the blood viscoelastic properties, such as indirect conventional techniques; thromboelastrograph (TEG) and rotational thromboelastometry (ROTEM) as well as recently proposed approaches, e.g., magnetomotive optical coherence elastography (Ahmad et al., 2014; Crecea et al., 2013) and laser speckle rheology (Tripathi et al., 2014). Although TEG and ROTEM (Evans et al., 2008; Wikkelso et al., 2017) have been widely used in the clinic to evaluate blood coagulation in patients, their destructive nature prevents further complementary characterization on the clots. Also, these methods can possess poor sensitivity and repeatability (Da Luz et al., 2014). Recently reported optical methods for evaluating blood coagulation in real-time (e.g., magnetomotive optical coherence elastography) require contact with blood samples and cannot monitor the dynamic changes in viscoelastic properties during blood coagulation (Ahmad et al., 2014; Crecea et al., 2013). As another example, non-contact laser speckle rheology lacks the ability to directly calculate the elastic modulus without subsequent calibration (Tripathi et al., 2014).

More recently, a nondestructive and contactless method has been proposed, which uses mechanical vibrations to accurately measure the rheological properties of soft biomaterials in real-time (Ceccaldi et al., 2017). This technique has been already tested and used in the agri-food industry (e.g., milk coagulation), and in the life science industry for

\footnotetext{
* Corresponding author.

E-mail address: showan.nazhat@mcgill.ca (S.N. Nazhat).
} 
(a)

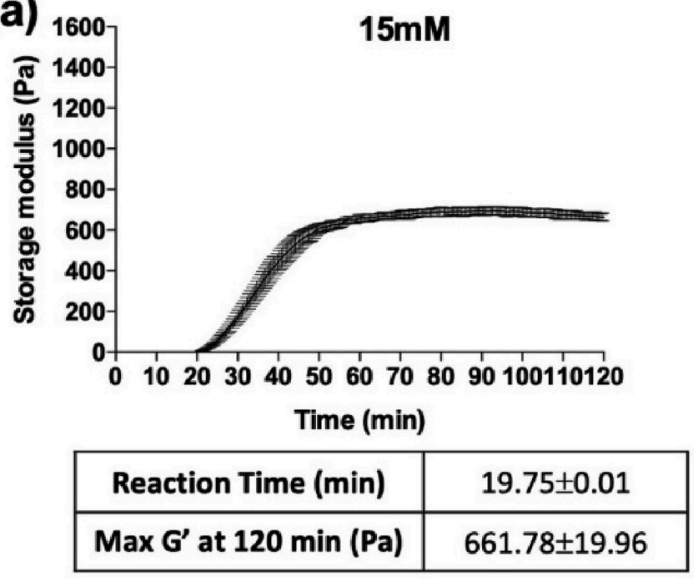

(c)

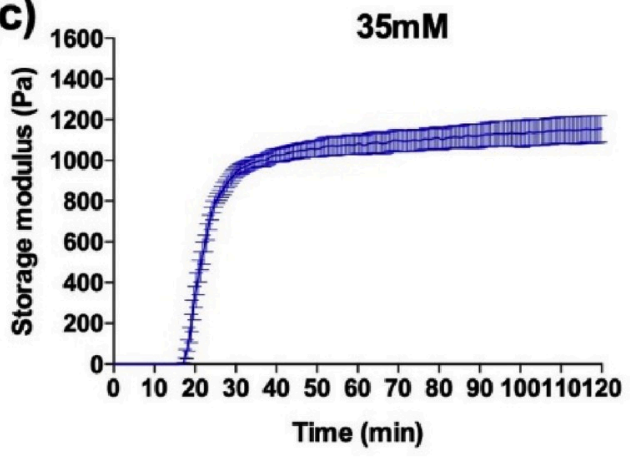

(b)

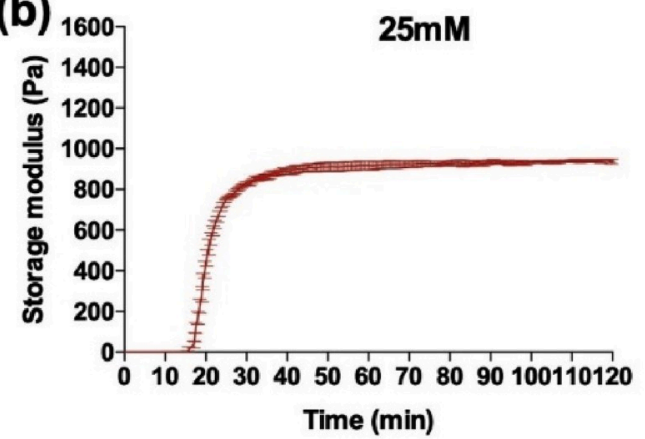

\begin{tabular}{|c|c|}
\hline Reaction Time $(\mathbf{m i n})$ & $15.72 \pm 0.19$ \\
\hline Max $\mathbf{G}^{\prime}$ at $120 \mathrm{~min}(\mathrm{~Pa})$ & $936.29 \pm 0.29$ \\
\hline
\end{tabular}

(d)

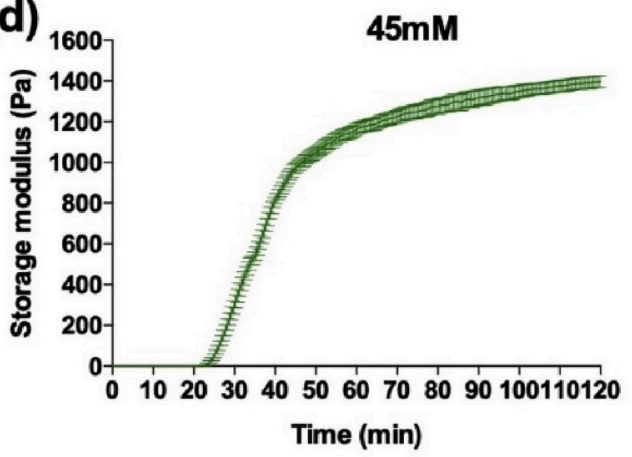

\begin{tabular}{|c|c|}
\hline Reaction Time $(\mathrm{min})$ & $22.72 \pm 0.22$ \\
\hline Max $\mathbf{G}^{\prime}$ at $120 \mathrm{~min}(\mathrm{~Pa})$ & $1399.17 \pm 29.17$ \\
\hline
\end{tabular}

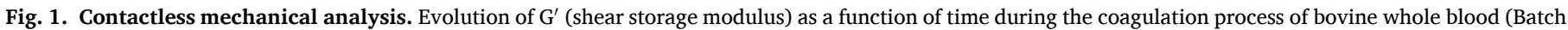
B) after recalcification with (a) 15, (b) 25 , (c) 35 and (d) $45 \mathrm{mM} \mathrm{CaCl}_{2}$ (Error bars: standard deviation: SD, $\mathrm{n}=3$ ).

biomaterials such as hydrogels (https://www.rheolution.com; Mirani et al., 2019). The goal of this study was to use this novel technique to quantitatively measure viscoelastic properties of whole blood in real-time, when exposed to different concentrations of calcium chloride $\left(\mathrm{CaCl}_{2}\right)$ and validate the outputs with already established techniques; TEG, a clinically used technique, and a conventional rheometer, which provided quantitative data on viscoelastic parameters.

\section{Experimental section}

\subsection{Materials}

Bovine whole blood (Sterile Sodium Citrate, CL1700-500C) was purchased from Cedarlane (Canada). Since the lifetime of each ordered batch was approximately two weeks, two different batches (Batch A and B) were utilized in this study to obtain the data. $\mathrm{CaCl}_{2}$ (Sigma-Aldrich, anhydrous, ACS reagent, $\geq 96 \%$ ) was used as received.

\subsection{Assessment of the blood coagulation}

The Elastosens ${ }^{\mathrm{TM}} \mathrm{Bio}^{2}$ (Rheolution Inc., Canada) instrument was used to examine the viscoelastic properties of blood. To measure the shear storage modulus $\left(\mathrm{G}^{\prime}\right)$ of a sample, a low frequency-amplitude (gentle mechanical) vibration is applied to the rigid sample holder, which also has a flexible, bottom membrane of silicone rubber. Next, the dynamic response of the sample is measured using a contactless laser probe and processed using specialized models.

In this study, the real-time changes in $\mathrm{G}^{\prime}$ of bovine whole blood after recalcification with $15,25,35$, and $45 \mathrm{mM} \mathrm{CaCl}_{2}$ were measured. Citrated bovine blood $(4.5 \mathrm{~mL})$ was initially placed in an incubator for $30 \mathrm{~min}$ at $37^{\circ} \mathrm{C}$. To activate coagulation, the blood sample was recalcified by adding $0.5 \mathrm{~mL}$ of the different concentrations of $\mathrm{CaCl}_{2}$ solutions. After 10 gentle vial inversions, the mixed whole blood was immediately loaded into a cylindrically shaped sample holder composed of a polycarbonate surrounding that is attached to a silicone rubber bottom surface by a dedicated adhesive. Prior to sample loading, the sample holders were washed with ethanol and placed in the instrument at $37^{\circ} \mathrm{C}$ for $30 \mathrm{~min}$. $\mathrm{G}^{\prime}$ of the blood samples was quantified over $120 \mathrm{~min}$ (with a 30 -second resolution) as an indication of coagulation. The resulting $\mathrm{G}^{\prime}$-time data were used to evaluate the reaction time (and can be related to activated clotting time) for each system, which was considered as the time at which $\mathrm{G}^{\prime}$ rapidly increased. Additionally, the clotting behaviour of citrated bovine whole blood with no $\mathrm{CaCl}_{2}$ was investigated as negative control and no significant effect of sample holder on coagulation was observed.

The profile of $\mathrm{G}^{\prime}$ versus time as a function of different concentrations of $\mathrm{CaCl}_{2}$ (i.e., 15, 25, 35, and $45 \mathrm{mM} \mathrm{CaCl}_{2}$ ) generated by the Elastosens ${ }^{\mathrm{TM}} \mathrm{Bio}^{2}$ was compared against the outputs of two conventionally used techniques; a TEG and a rheometer. TEG measurements were conducted according to the manufacturer's specifications using the Thromboelastrograph Model 5000 (Haemoscope Corporation, USA) in native mode at $37^{\circ} \mathrm{C}$ for $40 \mathrm{~min}$. On the TEG, the clotting time (reaction time), which is the time of latency from the start of the test to initial fibrin formation (the time from the start of a sample run until the first significant levels of detectable clot formation (amplitude $=2 \mathrm{~mm}$ )), and the Maximum Amplitude representing the ultimate strength of the fibrin 
(a)

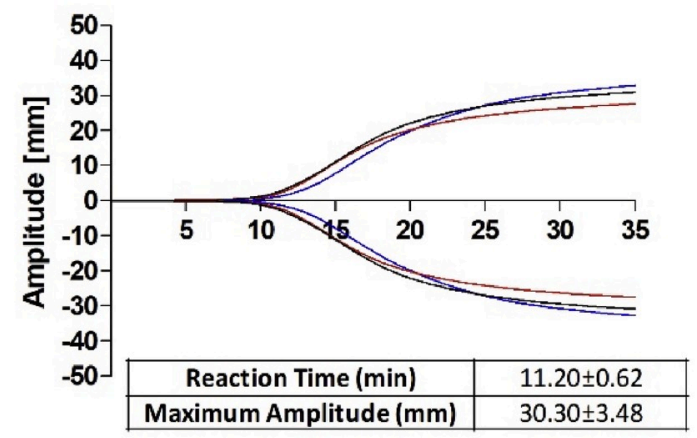

(c)

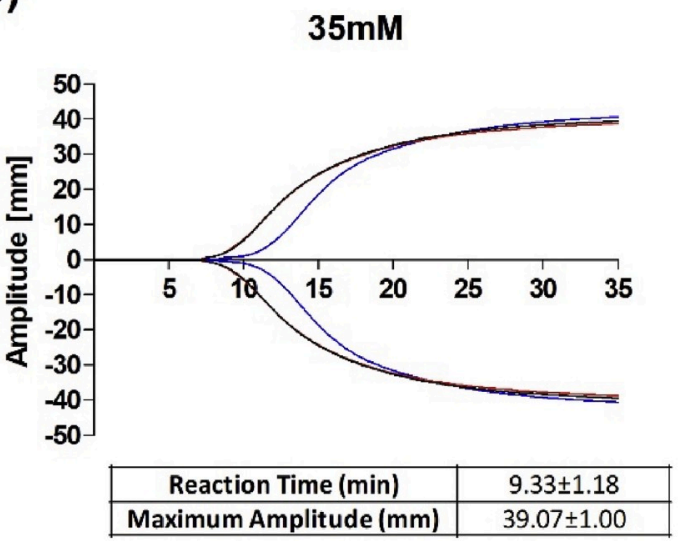

(b)
$25 \mathrm{mM}$

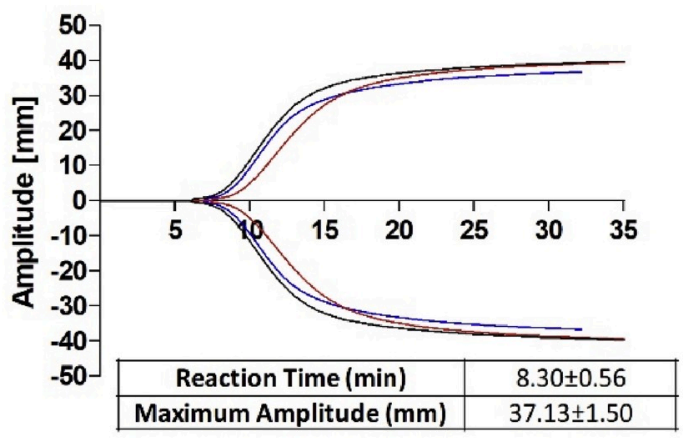

(d)

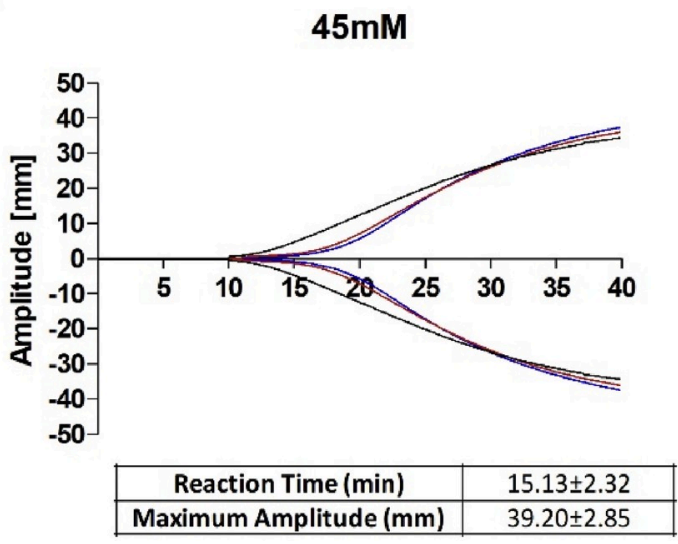

Fig. 2. Thromboelastrograph (TEG) analysis. A depiction of the TEG output for bovine whole blood (Batch A) after recalcification with different $\mathrm{CaCl}_{2}$ concentrations of (a)15, (b) 25 , (c) 35 and (d) $45 \mathrm{mM}$.

clot, were calculated for each measurement (Whiting and DiNardo, 2014). In the case of the rheometer (MCR 302 Anton Paar, Austria), 0.65 $\mathrm{mL}$ of citrated bovine whole blood post recalcification, was loaded on the rheometer stage with $25 \mathrm{~mm}$ diameter stainless steel parallel plate geometry and gap height was adjusted at $0.8 \mathrm{~mm}$. To avoid blood dehydration during the test, an inert solvent trap was applied after trimming the loaded sample. Measurements were conducted under a constant oscillation frequency of $1.5 \mathrm{~Hz}$ and a deformation amplitude of $0.1 \%$ with 30 -second resolution. The $\mathrm{G}^{\prime}$ of the samples was measured at $37{ }^{\circ} \mathrm{C}$ over $120 \mathrm{~min}$. The reaction time was defined as the time when $\mathrm{G}^{\prime}$ was $\geq 10 \mathrm{~Pa}$.

\subsection{Statistical analysis}

Statistical significance of the measured parameters between samples was determined using the Student's t-test at a significance level of $\mathrm{p}<$ 0.05 .

\section{Results and discussion}

The Elastosens ${ }^{\mathrm{TM}} \mathrm{Bio}^{2}$ instrument was used to examine the real-time changes in shear storage modulus (i.e., $\mathrm{G}^{\prime}$ ) of blood during the coagulation process. To evaluate the reproducibility of the instrument, triplicate measurements were initially performed at three different days; 1 , 2 and 7 where the changes in $\mathrm{G}^{\prime}$ of the whole blood (Batch A) were monitored after recalcification with $45 \mathrm{mM} \mathrm{CaCl}_{2}$ (Supplementary Material; Fig. S1). The rapid increase in $\mathrm{G}^{\prime}$ for all the curves occurred at the same time followed by similar increasing rates up to their maximum $\mathrm{G}^{\prime}$ (plateau) of the same range (Fig. S1). Based on these results, it was confirmed that the measured values were consistent over time, and that the instrument was able to accurately monitor changes in blood clot biomechanics.

To further investigate the precision of this technique, the Elastosens ${ }^{\mathrm{TM}} \mathrm{Bio}^{2}$ was used to measure the effect of different concentrations of $\mathrm{CaCl}_{2}$ on the real-time temporal changes in $\mathrm{G}^{\prime}$. Fig. 1 shows the changes in $\mathrm{G}^{\prime}$ of whole blood (Batch B) after recalcification with 15, 25, 35 and $45 \mathrm{mM} \mathrm{CaCl}_{2}$. According to this figure, increasing the $\mathrm{CaCl}_{2}$ concentration from 15 to $25 \mathrm{mM}$ decreased the reaction time (i.e., shorter clotting time). However, a further increase in the salt concentration to 35 and $45 \mathrm{mM}$ favored longer clotting times. In fact, it has been discussed that $\mathrm{CaCl}_{2}$ can be used as an anticoagulant at higher concentrations (Giraldo et al., 2017; Crawford et al., 1987; Mikaelsson, 1991). In relation to the maximum $\mathrm{G}^{\prime}$ as an indication of clot stiffness, higher amounts of $\mathrm{CaCl}_{2}$ resulted in higher shear storage modulus values, suggesting the clots were stiffer (Tran et al., 2013). The final blood clots were further characterized using scanning electron microscopy (Supplementary Material; Fig. S2) to evaluate the feasibility of post processing characterization of blood clot samples in this non-destructive testing approach, which is not possible in traditional methods. It was observed that the morphology of red blood cells was preserved in blood clots after the measurement by Elastosens ${ }^{\mathrm{TM}} \mathrm{Bio}^{2}$ (Dibiasi et al., 2018; Kawasaki et al., 2004).

Fig. 2 presents the TEG output of whole blood (Batch A) in the presence of $15,25,35$ and $45 \mathrm{mM} \mathrm{CaCl}_{2}$ concentrations. TEG is a conventional method for clinically evaluating blood coagulation properties (Whiting and DiNardo, 2014; Cahill et al., 2018). It was demonstrated that, at the lower concentrations, the higher the calcium salt, the shorter the reaction (clotting) times whereas at the higher concentrations, the 


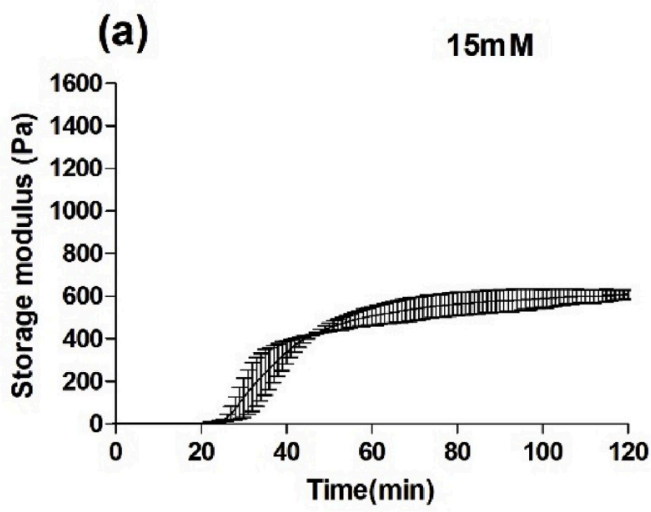

\begin{tabular}{|c|c|}
\hline Reaction Time (min) & $24.50 \pm 0.71$ \\
\hline Max G'at $120 \mathrm{~min}(\mathrm{~Pa})$ & $606.65 \pm 21.38$ \\
\hline
\end{tabular}

(c)

$35 \mathrm{mM}$

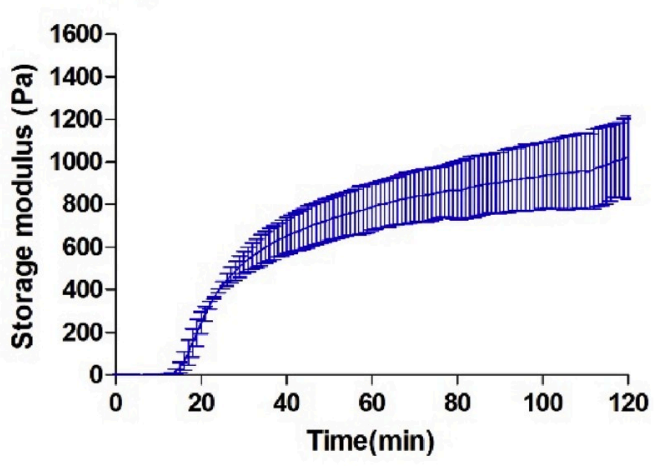

\begin{tabular}{|c|c|}
\hline Reaction Time (min) & $14.50 \pm 0.71$ \\
\hline Max G'at 120 min (Pa) & $1010.71 \pm 195.58$ \\
\hline
\end{tabular}

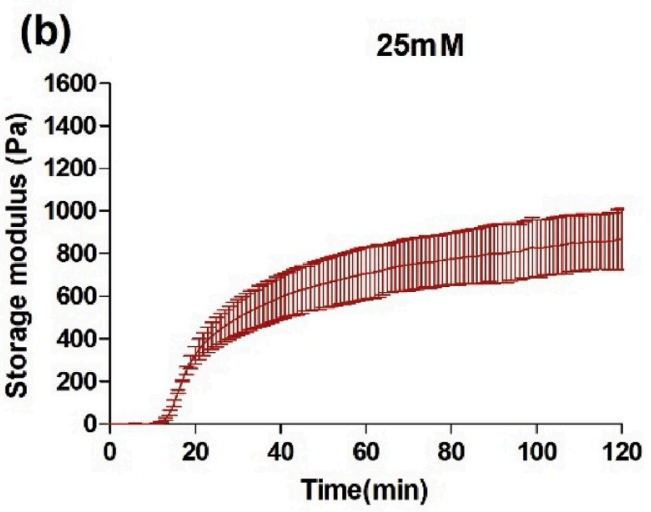

\begin{tabular}{|c|c|}
\hline Reaction Time (min) & $13.00 \pm 0.00$ \\
\hline Max G’at 120 min (Pa) & $866.38 \pm 140.10$ \\
\hline
\end{tabular}

(d)

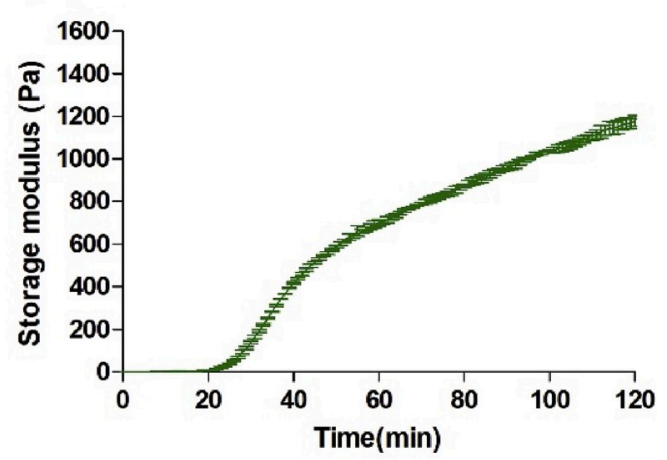

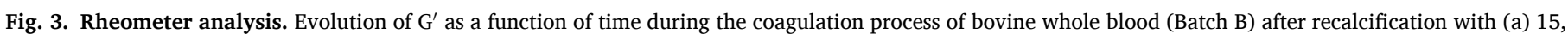
(b) 25, (c) 35 and (d) $45 \mathrm{mM} \mathrm{CaCl}_{2}$ (Error bars: standard deviation: SD, $\mathrm{n}=3$ ).

higher the calcium salt, the longer the reaction times. While the results are consistent with those of the Elastosens ${ }^{\mathrm{TM}} \mathrm{Bio}^{2}$, the qualitative Maximum Amplitude parameter is not as informative as the $\mathrm{G}^{\prime}$ values and clot stiffness. Furthermore, there was no statistically significant difference ( $p>0.05$ ) between the Maximum Amplitude values of the samples with 25,35 and $45 \mathrm{mM} \mathrm{CaCl}_{2}$ concentrations. This suggests that TEG lacks the ability to quantitatively measure blood clot biomechanics with potentially poor sensitivity and repeatability, as indicated by the higher error values.

Furthermore, in this study, a rheometer was utilized to validate the evolution of $\mathrm{G}^{\prime}$ values of the blood measured by Elastosens ${ }^{\mathrm{TM}} \mathrm{Bio}^{2}$. Rheometers are well-known tools to accurately measure the viscoelastic properties of materials, such as biopolymer hydrogels (Day, 2005; Matos et al., 2019). Fig. 3 shows the shear storage modulus of whole blood (Batch $\mathrm{B}$ ) in the presence of different $\mathrm{CaCl}_{2}$ concentrations of $15,25,35$ and $45 \mathrm{mM}$. It was shown that the stiffness of the clots (i.e., the maximum $\mathrm{G}^{\prime}$ of the blood samples) increased with increasing calcium concentration, within the same range as those measured by Elastosens ${ }^{\mathrm{TM}}$ $\mathrm{Bio}^{2}$. Interestingly, the blood sample recalcified with $45 \mathrm{mM} \mathrm{CaCl}$ did not reach a stable value, a finding that requires further investigation.

\subsection{Correlation between different sets of values and $\mathrm{CaCl}_{2}$ concentrations}

The reaction time (Fig. 4a), as well as storage modulus and maximum amplitude (Fig. 4b) values obtained from various techniques as a function of $\mathrm{CaCl}_{2}$ concentrations were correlated. The trend in Fig. $4 \mathrm{a}$ is in great agreement with previous studies (Mikaelsson, 1991) verifying the ability of this contactless instrument in accurately evaluating the clotting parameters of blood for research and clinical applications. Furthermore, the direct correlation between the shear modulus data (Fig. 4c) generated through the Elastosens ${ }^{\mathrm{TM}} \mathrm{Bio}^{2}$ and the rheometer quantitatively confirmed the accuracy of the non-destructive technique in measuring blood viscoelastic properties. Table S1 provides the coefficient of variation of the mean values of the data generated from the various techniques.

\section{Conclusion}

This study presented a novel approach to accurately and quantitatively measure and monitor the real-time changes in blood coagulation viscoelastic properties using a contactless, non-destructive technique (Elastosens ${ }^{\mathrm{TM}} \mathrm{Bio}^{2}$ ). The effect of $\mathrm{CaCl}_{2}$ concentration on the clotting time and storage modulus of the developed clots was measured and validated with those generated by conventional instruments (TEG and rheometer). The results revealed that increasing the calcium concentration from low values shortened the clotting times, whereas a further increase in calcium concentration led to longer reaction times. Also, increasing the $\mathrm{CaCl}_{2}$ concentration resulted in higher maximum $\mathrm{G}^{\prime}$, which has been associated with the formation of stiffer clots.

Ultimately, it was demonstrated that the Elastosens ${ }^{\mathrm{TM}} \mathrm{Bio}^{2}$ offers a 
(a)

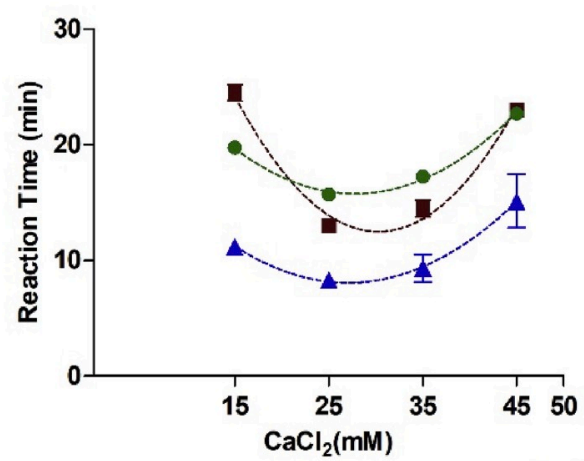

(b)

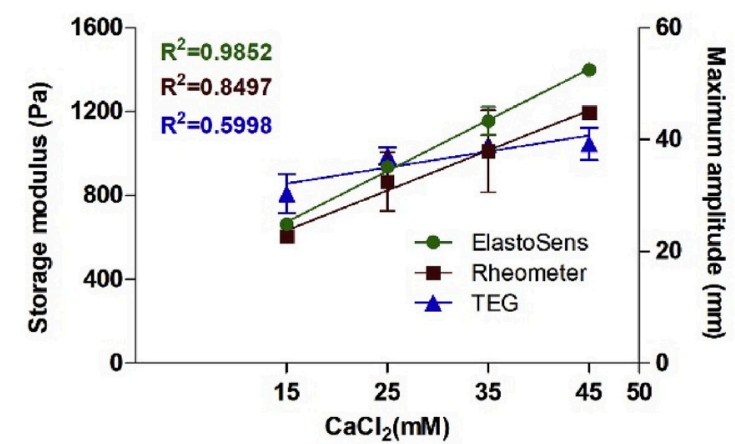

(c)

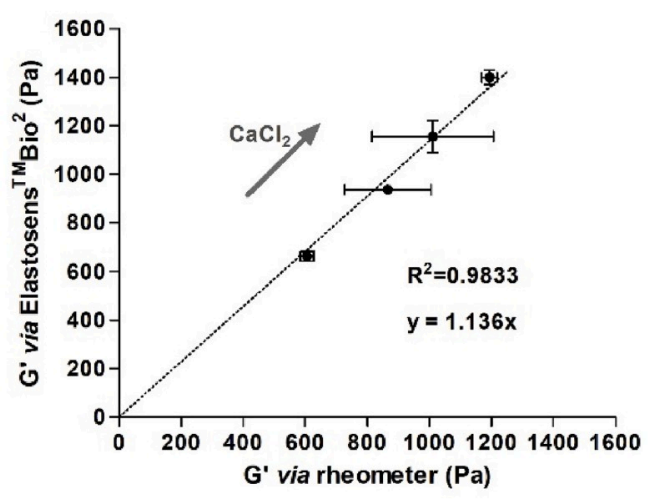

Fig. 4. Correlation between (a) reaction time and $\mathrm{CaCl}_{2}$ concentration, (b) storage modulus or maximum amplitude and $\mathrm{CaCl}_{2}$ concentration, measured by Elastosens ${ }^{\mathrm{TM}} \mathrm{Bio}^{2}$, rheometer, and TEG (c) Linear correlation between storage modulus values measured by Elastosens ${ }^{\mathrm{TM}}$ Bio $^{2}$ and rheometer (Error bars: standard deviation: $\mathrm{SD}, \mathrm{n}=3$ ).

promising tool that can provide a relevant combination of data for assessing the efficacy of hemostatic agents as well as for clinicians to better understand, predict, diagnose, and eventually treat blood related diseases. For many reasons, the use of different hemostatic agents varies between countries and surgeons. Despite the need, there is no comprehensive, detailed guideline for the selection of appropriate hemostatic agents for different conditions, mostly due to the scarcity of the relevant published literature (Barnard and Millner, 2009; Bracey et al., 2017). Considering the increasing number of studies on hemostatic agents (Tran et al., 2013; Barnard and Millner, 2009; Bracey et al., 2017), including (mesoporous) bioactive glasses (Pourshahrestani et al., 2016), and their functionality and characteristics, this contactless, non-destructive technique can provide an efficient approach for accurately assessing the influence of these materials on the blood coagulation characteristics. However, given that this approach is new, further research is needed to optimize the measurement parameters (e.g., sample volume, temperature, exposure time, and anti- and procoagulants concentration, etc.) and to develop universal protocols for various blood coagulation related concerns in line with already approved methods.

\section{Declaration of competing interest}

The authors declare that they have no known competing financial interests or personal relationships that could have appeared to influence the work reported in this paper.

\section{CRediT authorship contribution statement}

Shiva Naseri: Conceptualization, Methodology, Validation, Investigation, Visualization, Writing - original draft. Newsha Koushki:
Methodology, Validation, Investigation, Writing - review \& editing. Ehsan Rezabeigi: Validation, Writing - review \& editing. Allen Ehrlicher: Validation, Supervision, Resources, Funding acquisition, Writing - review \& editing. Showan N. Nazhat: Conceptualization, Validation, Supervision, Resources, Funding acquisition, Writing - review \& editing.

\section{Acknowledgements}

Funding for this research was provided by NSERC, EGP/5288152018 and McGill University. Rheometer was provided by NSERC EQPEQ/472339-2015 fund. The authors would like to thank Rheolution Inc. (Montreal, Canada) for providing access to their Elastosens ${ }^{\mathrm{TM}} \mathrm{Bio}^{2}$ device to perform the rheological experiments.

\section{Appendix A. Supplementary data}

Supplementary data to this article can be found online at https://doi. org/10.1016/j.jmbbm.2020.103921.

\section{References}

Ahmad, A., Kim, J., Sobh, N.A., Shemonski, N.D., Boppart, S.A., 2014. Magnetomotive optical coherence elastography using magnetic particles to induce mechanical waves. Biomed. Optic Express 5, 2349-2361.

Barnard, J., Millner, R., 2009. A review of topical hemostatic agents for use in cardiac surgery. Ann. Thorac. Surg. 88, 1377-1383.

Berglin, M., Andersson, M., Sellborn, A., Elwing, H., 2004. The effect of substrate molecular mobility on surface induced immune complement activation and blood plasma coagulation. Biomaterials 25, 4581-4590.

Bracey, A., Shander, A., Aronson, S., Boucher, B.A., Calcaterra, D., Chu, M.W., Culbertson, R., Jabr, K., Kehlet, H., Lattouf, O., Malaisrie, S.C., et al., 2017. The use of topical hemostatic agents in cardiothoracic surgery. Ann. Thorac. Surg. 104, 353-360. 
Cahill, C., Sharma, A., Blumberg, N., Schmidt, A.E., Refaai, M.A., 2018. Utilizing thromboelastography (TEG) as a bleeding risk assessment tool prior to interventional radiology procedures. Transfusion 58, 38a-38a.

Ceccaldi, C., Strandman, S., Hui, E., Montagnon, E., Schmitt, C., Hadj Henni, A., Lerouge, S., 2017. Validation and application of a nondestructive and contactless method for rheological evaluation of biomaterials. J. Biomed. Mater. Res. B Appl. Biomater. 105, 2565-2573.

Crawford, N., Crook, M., Dawes, J., Gray, C.R.W., 1987. The sole use of magnesiumchloride for blood anticoagulation - preservation of platelet surface-bound proteinaceous halo. Thromb. Haemostasis 58, 245-245.

Crecea, V., Ahmad, A., Boppart, S.A., 2013. Magnetomotive optical coherence elastography for microrheology of biological tissues. J. Biomed. Optic. 18.

Da Luz, L.T., Nascimento, B., Shankarakutty, A.K., Rizoli, S., Adhikari, N.K.J., 2014 Effect of thromboelastography (TEG (R)) and rotational thromboelastometry (ROTEM (R)) on diagnosis of coagulopathy, transfusion guidance and mortality in trauma: descriptive systematic review. Crit. Care 18, 518-544.

Day, C., 2005. Single, physics-based model accounts for the mechanical properties of diverse biopolymer gels. Phys. Today 58, 27-29.

Dibiasi, C., Plewka, J., Ploszczanski, L., Glanz, V., Lichtenegger, H., Windberger, U., 2018. Viscoelasticity and structure of blood clots generated in-vitro by rheometry: a comparison between human, horse, rat, and camel. Clin. Hemorheol. Microcirc. 69, 515-531.

Evans, P.A., Hawkins, K., Lawrence, M., Williams, R.L., Barrow, M.S., Thirumalai, N., Williams, P.R., 2008. Rheometry and associated techniques for blood coagulation studies. Med. Eng. Phys. 30, 671-679.

Giraldo, C.E., Alvarez, M.E., Carmona, J.U., 2017. Influence of calcium salts and bovine thrombin on growth factor release from equine platelet-rich gel supernatants. Vet. Comp. Orthop. Traumatol. 30, 1-7.

Guillin, M.C., Bezeoud, A., 1985. The blood coagulation process and its regulation. I. general mechanisms of blood coagulation. Pathol. Biol. 33, 847-857.

https://www.rheolution.com.

Kawasaki, J., Katori, N., Kodaka, M., Miyao, H., Tanaka, K.A., 2004. Electron microscopic evaluations of clot morphology during Thrombelastography((R)). Anesth. Analg. 99, 1440-1444.
Matos, B.D.M., Rocha, V., da Silva, E.J., Moro, F.H., Bottene, A.C., Ribeiro, C.A., dos Santos Dias, D., Antonio, S.G., do Amaral, A.C., Cruz, S.A., de Oliveira Barud, H.G., Silva Barud, H., 2019. Evaluation of commercially available polylactic acid (PLA) filaments for 3D printing applications. J. Therm. Anal. Calorim. 137, 555-562.

Mikaelsson, M.E., 1991. The role of calcium in coagulation and anticoagulation. Coagulation and Blood Transfusion 26, 29-37.

Mirani, B., Pagan, E., Shojaei, S., Duchscherer, J., Toyota, B.D., Ghavami, S., Akbari, M., 2019. A 3D bioprinted hydrogel mesh loaded with all-trans retinoic acid for treatment of glioblastoma. Eur. J. Pharmacol. 854, 201-212.

Peyvandi, F., Jayandharan, G., Chandy, M., Srivastava, A., Nakaya, S.M., Johnson, M.J., Thompson, A.R., Goodeve, A., Garagiola, I., Lavoretano, S., Menegatti, M., Palla, R., Spreafico, M., Tagliabue, L., Asselta, R., Duga, S., Mannucci, P.M., 2006. Genetic diagnosis of haemophilia and other inherited bleeding disorders. Haemophilia 12 82-89.

Pourshahrestani, S., Zeimaran, E., Kadri, N.A., Gargiulo, N., Samuel, S., Naveen, S.V., Kamarul, T., Towler, M.R., 2016. Gallium-containing mesoporous bioactive glass with potent hemostatic activity and antibacterial efficacy. J. Mater. Chem. B 4, 71-86.

Tanaka, M., Sato, K., Kitakami, E., Kobayashi, S., Hoshiba, T., Fukushima, K., 2015. Design of biocompatible and biodegradable polymers based on intermediate water concept. Polym. J. 47, 114-121.

Tran, R., Myers, D.R., Ciciliano, J., Trybus Hardy, E.L., Sakurai, Y., Ahn, B., Qiu, Y., Mannino, R.G., Fay, M.E., Lam, W.A., 2013. Biomechanics of haemostasis and thrombosis in health and disease: from the macro- to molecular scale. J. Cell Mol. Med. 17, 579-596.

Tripathi, M.M., Hajjarian, Z., Van Cott, E.M., Nadkarni, S.K., 2014. Assessing blood coagulation status with laser speckle rheology. Biomed. Optic Express 5, 817-831.

Weisel, J.W., 2010. Biomechanics in hemostasis and thrombosis. J. Thromb. Haemostasis 8, 1027-1029.

Whiting, D., DiNardo, J.A., 2014. TEG and ROTEM: Technology and clinical applications. Am. J. Hematol. 89, 228-232.

Wikkelso, A., Wetterslev, J., Moller, A.M., Afshari, A., 2017. Thromboelastography (TEG) or rotational thromboelastometry (ROTEM) to monitor haemostatic treatment in bleeding patients: a systematic review with meta-analysis and trial sequential analysis. Anaesthesia 72, 519-531. 